Adv. Studies Theor. Phys., Vol. 7, 2013, no. 3, 121 - 126 HIKARI Ltd, www.m-hikari.com

\title{
Computational Experiments for the Problem of Hamiltonian Path with Fixed Number of Color Repetitions
}

\author{
Anna Gorbenko \\ Department of Intelligent Systems and Robotics \\ Ural Federal University \\ 620083 Ekaterinburg, Russia \\ gorbenko.ann@gmail.com \\ Vladimir Popov \\ Department of Intelligent Systems and Robotics \\ Ural Federal University \\ 620083 Ekaterinburg, Russia \\ Vladimir.Popov@usu.ru
}

\begin{abstract}
In this paper we consider an approach to solve the problem of Hamiltonian path with fixed number of color repetitions for arc-colored digraphs. Our approach is based on usage of local search algorithms to solve a logical model for the problem.
\end{abstract}

PACS: 07.05.Fb, 07.05.Dz

Keywords: Hamiltonian path, arc-colored digraph, satisfiability problem, NP-complete

Let $G=(V ; E)$ be a digraph where

$$
V=\{v[1], v[2], \ldots, v[n]\}
$$

is the set of nodes,

$$
E=\{(v[i[1]], v[j[1]]), \ldots\}
$$

is the set of arcs. We assume that each arc of $G$ has a color. If the number of colors of arcs is restricted by an integer $c$, we speak about $c$-arc-colored 
digraphs. Let $C[(u, v)]$ be the color of $(u, v)$. We can assume that

$$
C[(u, v)] \in\{1,2, \ldots, c\} .
$$

Let $r$ be a fixed positive integer. Now, we consider the following problem.

The PROBlem of HAMILTONIAN PATH WITH FIXED NUMBER OF COLOR REPETITIONS FOR $c$-ARC-COLORED DIGRAPHS:

InstancE: A c-arc-colored digraph $G=(V ; E)$.

QUESTION: Is $G$ has a Hamiltonian path

$$
v[k[0]], v[k[1]], \ldots, v[k[n-1]]
$$

such that

$$
\begin{gathered}
C[(v[k[i]], v[k[i+1]])]= \\
C[(v[k[i+1]], v[k[i+2]])]=\ldots=C[(v[k[i+r-1]], v[k[i+r]])], \\
|C[(v[k[j]], v[k[j+1]])]-C[(v[k[j+1]], v[k[j+2]])]|>0,
\end{gathered}
$$

for all

$$
\begin{gathered}
i=0 \bmod r, \\
j+1=0 \bmod r, \\
i \in\{0,1, \ldots, n-1\}, \\
j \in\{0,1, \ldots, n-1\} ?
\end{gathered}
$$

Note that different Hamiltonian path problems are extensively studied (see e.g. [1] - [3]). have natural applications in the design of experiments. In particular, the problem of Hamiltonian path with fixed number of color repetitions for $c$-arc-colored digraphs can be used for scenarios creation for self-learning of intelligent mobile robots.

Note that the problem of Hamiltonian path with fixed number of color repetitions for $c$-arc-colored digraphs is NP-complete. Encoding different hard problems as instances of SAT and solving them with efficient satisfiability algorithms has caused considerable interest (see e.g. [4] - [19]). In this paper, we consider an approach to solve the problem of Hamiltonian path with fixed number of color repetitions for $c$-arc-colored digraphs. Our approach is based on an explicit reduction from the problem to the satisfiability problem. We consider different local search algorithms to solve a logical model for the problem.

Let

$$
\begin{aligned}
\varphi[1]= & \wedge_{0 \leq i \leq n-1} \vee_{1 \leq j \leq n} x[i, j], \\
\varphi[2]= & \wedge_{0 \leq i \leq n-1, \quad} \quad(\neg x[i, j[1]] \vee \neg x[i, j[2]]), \\
& 1 \leq j[1]<j[2] \leq n
\end{aligned}
$$




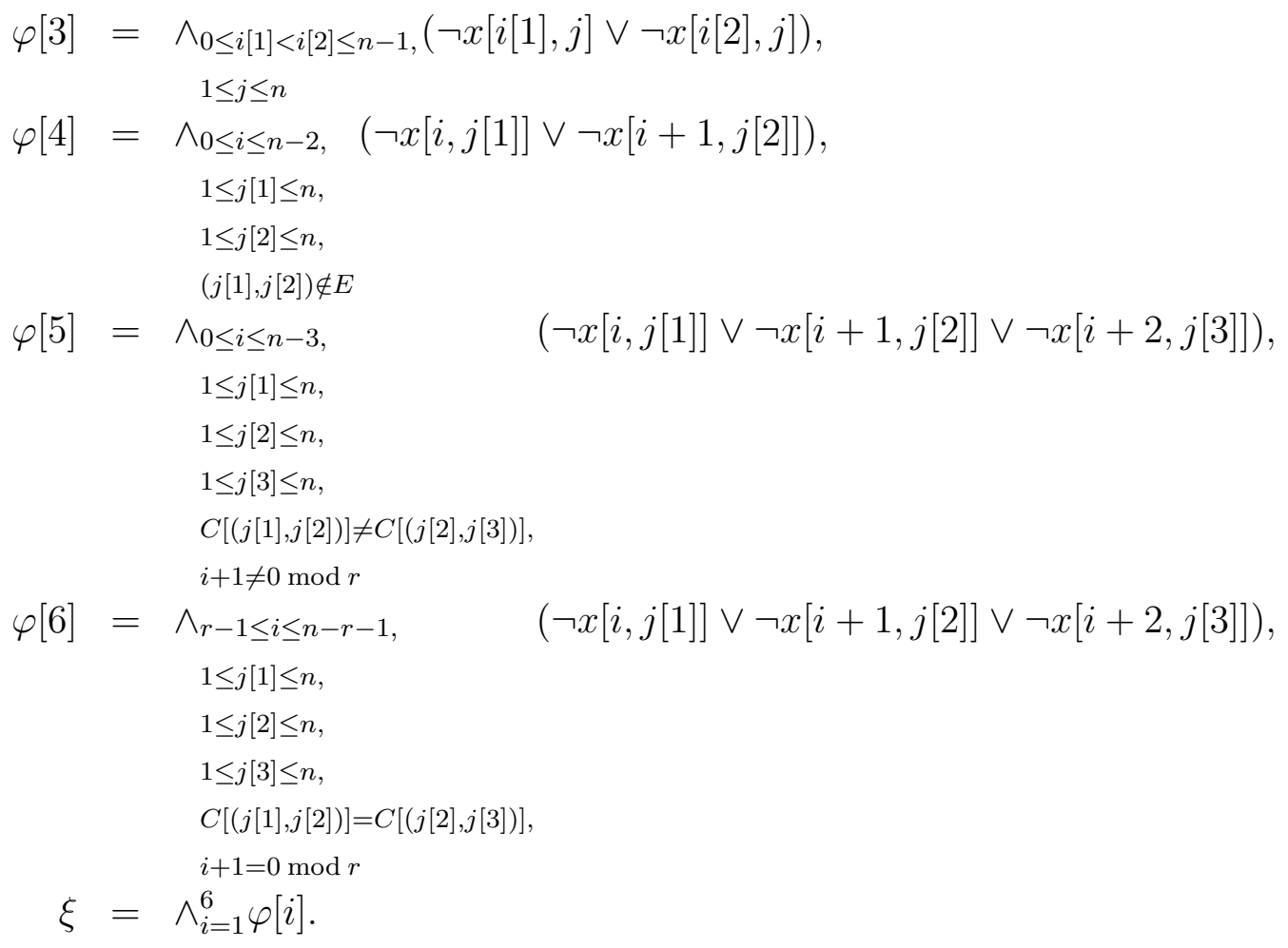

Theorem. The function $\xi$ is satisfiable if and only if there is a Hamilton path

$$
v[k[0]], v[k[1]], \ldots, v[k[n-1]]
$$

in $G$ such that

$$
\begin{gathered}
C[(v[k[i]], v[k[i+1]])]= \\
C[(v[k[i+1]], v[k[i+2]])]=\ldots=C[(v[k[i+r-1]], v[k[i+r]])], \\
|C[(v[k[j]], v[k[j+1]])]-C[(v[k[j+1]], v[k[j+2]])]|>0,
\end{gathered}
$$

for all

$$
\begin{gathered}
i=0 \bmod r, \\
j+1=0 \bmod r, \\
i \in\{0,1, \ldots, n-1\}, \\
j \in\{0,1, \ldots, n-1\} .
\end{gathered}
$$

Proof. Let $\xi=1$. In view of the definition of $\xi$, in this case, it is clear that $\varphi[i]=1$, for all $1 \leq i \leq 6$.

Since $\varphi[1]=1$, it is easy to see that for all $0 \leq i \leq n-1$, there is $1 \leq j \leq n$ such that $x[i, j]=1$. In view of $\varphi[2]=1$, it is clear that for any $0 \leq i \leq n-1$, there is only one value of $j$ such that $x[i, j]=1$. Let $x[i, a[i]]=1$. Since $\varphi[3]=1$, it is easy to see that $a[i] \neq a[j]$, for any $i \neq j$. Therefore, values of 
$x[i, j]$ can be considered as a choice of nodes for a path $y[0], y[1], \ldots, y[n-1]$. In particular, we can assume that $v[j]$ is the $i$ th node in the path if and only if $x[i, j]=1$.

Clearly, $\varphi[4]=1$ if and only if the sequence

$$
y[0], y[1], \ldots, y[n-1]
$$

of nodes is a path. Since $\varphi[5]=1$, it is easy to see that

$$
C[(y[i], y[i+1])]=C[(y[i+1], y[i+2])]=\ldots=C[(y[i+r-1], y[i+r])],
$$

for all

$$
\begin{gathered}
i=0 \bmod r, \\
i \in\{0,1, \ldots, n-1\} .
\end{gathered}
$$

In view of $\varphi[6]=1$, we obtain that

$$
\begin{gathered}
|C[(y[j], y[j+1])]-C[(y[j+1], y[j+2])]|>0, \\
j+1=0 \bmod r, \\
j \in\{0,1, \ldots, n-1\} .
\end{gathered}
$$

Now, we assume that there is a Hamilton path

$$
v[k[0]], v[k[1]], \ldots, v[k[n-1]]
$$

in $G$ such that

$$
\begin{gathered}
C[(v[k[i]], v[k[i+1]])]= \\
C[(v[k[i+1]], v[k[i+2]])]=\ldots=C[(v[k[i+r-1]], v[k[i+r]])], \\
|C[(v[k[j]], v[k[j+1]])]-C[(v[k[j+1]], v[k[j+2]])]|>0,
\end{gathered}
$$

for all

$$
\begin{gathered}
i=0 \bmod r, \\
j+1=0 \bmod r, \\
i \in\{0,1, \ldots, n-1\}, \\
j \in\{0,1, \ldots, n-1\} .
\end{gathered}
$$

Let $x[i, j]=1$ if and only if $j=k[i]$, for all $0 \leq i \leq n-1$. It is easy to check that $\xi=1$.

Using standard transformations (see e.g. [20]) we can easily obtain an explicit transformation $\xi$ into $\zeta$ such that $\xi \Leftrightarrow \zeta$ and $\zeta$ is a 3 -CNF. It is clear that $\zeta$ gives us an explicit reduction from the problem of Hamiltonian path with fixed number of color repetitions for $c$-arc-colored digraphs to 3SAT. 
We have created a generator of natural instances for the problem of Hamiltonian path with fixed number of color repetitions for $c$-arc-colored digraphs. There is a well known site on which posted solvers for SAT [21]. We have used algorithms from [21]: fgrasp and posit. For solution of the problem, we have used a heterogeneous cluster. Each test was run on a cluster of at least 100 nodes. Note that due to restrictions on computation time (20 hours) we used savepoints. Selected experimental results are given in Table .

\begin{tabular}{|l|lll|}
\hline time & average & $\max$ & best \\
\hline fgrasp & $19.74 \mathrm{hr}$ & $56.04 \mathrm{hr}$ & $27.12 \mathrm{~min}$ \\
posit & $12.63 \mathrm{hr}$ & $37.25 \mathrm{hr}$ & $31.08 \mathrm{~min}$ \\
\hline
\end{tabular}

Table 1: Experimental results for 3SAT

ACKNOWLEDGEMENTS. The work was partially supported by Analytical Departmental Program "Developing the scientific potential of high school" 8.1616.2011.

\section{References}

[1] A. Gorbenko and V. Popov, The Problem of Finding Two Edge-Disjoint Hamiltonian Cycles, Applied Mathematical Sciences, 6 (2012), 6563-6566.

[2] A. Gorbenko and V. Popov, Hamiltonian Alternating Cycles with Fixed Number of Color Appearances, Applied Mathematical Sciences, 6 (2012), 6729-6731.

[3] A. Gorbenko and V. Popov, The Hamiltonian Alternating Path Problem, IAENG International Journal of Applied Mathematics, 42 (2012) 204-213.

[4] A. Gorbenko and V. Popov, Footstep Planning for Humanoid Robots, Applied Mathematical Sciences, 6 (2012), 6567-6571.

[5] A. Gorbenko, M. Mornev, V. Popov, and A. Sheka, The problem of sensor placement for triangulation-based localisation, International Journal of Automation and Control, 5 (2011) 245-253.

[6] A. Gorbenko and V. Popov, SAT Solvers for the Problem of Sensor Placement, Advanced Studies in Theoretical Physics, 6 (2012) 1235-1238.

[7] A. Gorbenko and V. Popov, Clustering Algorithm in Mobile Ad Hoc Networks, Advanced Studies in Theoretical Physics, 6 (2012) 1239-1242. 
[8] A. Gorbenko, M. Mornev, and V. Popov, Planning a Typical Working Day for Indoor Service Robots, IAENG International Journal of Computer Science, 38 (2011) 176-182.

[9] A. Gorbenko and V. Popov, The Problem of Selection of a Minimal Set of Visual Landmarks, Applied Mathematical Sciences, 6 (2012) 4729-4732.

[10] A. Gorbenko and V. Popov, On the Problem of Placement of Visual Landmarks, Applied Mathematical Sciences, 6 (2012) 689-696.

[11] A. Gorbenko and V. Popov, On the Longest Common Subsequence Problem, Applied Mathematical Sciences, 6 (2012), 5781-5787.

[12] A. Gorbenko and V. Popov, On the Problem of Sensor Placement, Advanced Studies in Theoretical Physics, 6 (2012) 1117-1120.

[13] A. Gorbenko and V. Popov, The Longest Common Parameterized Subsequence Problem, Applied Mathematical Sciences, 6 (2012), 2851-2855.

[14] A. Gorbenko and V. Popov, The set of parameterized k-covers problem, Theoretical Computer Science, 423 (2012), 19-24.

[15] A. Gorbenko and V. Popov, The Far From Most String Problem, Applied Mathematical Sciences, 6 (2012), 6719-6724.

[16] A. Gorbenko and V. Popov, Multi-agent Path Planning, Applied Mathematical Sciences, 6 (2012), 6733-6737.

[17] A. Gorbenko and V. Popov, Programming for Modular Reconfigurable Robots, Programming and Computer Software, 38 (2012) 13-23.

[18] A. Gorbenko, V. Popov, and A. Sheka, Localization on Discrete Grid Graphs, Lecture Notes in Electrical Engineering, 107 (2012), 971-978.

[19] A. Gorbenko and V. Popov, Task-resource Scheduling Problem, International Journal of Automation and Computing, 9 (2012), 429-441.

[20] A. Gorbenko and V. Popov, The c-Fragment Longest Arc-Preserving Common Subsequence Problem, IAENG International Journal of Computer Science, 39 (2012), 231-238.

[21] SATLIB — The Satisfiability Library. [Online]. Available: http://people.cs.ubc.ca/ hoos/SATLIB/index-ubc.html

\section{Received: December 3, 2012}

